\title{
On evaluating the performance of intentional controlled islanding schemes
}

DOI:

10.1109/PESMG.2013.6672423

Link to publication record in Manchester Research Explorer

\section{Citation for published version (APA):}

Quiros-Tortos, J., Panteli, M., Terzija, V., \& Crossley, P. A. (2013). On evaluating the performance of intentional controlled islanding schemes. In IEEE Power and Energy Society General Meeting/IEEE Power Energy Soc. Gen. Meet. IEEE. https://doi.org/10.1109/PESMG.2013.6672423

\section{Published in:}

IEEE Power and Energy Society General Meeting|IEEE Power Energy Soc. Gen. Meet.

\section{Citing this paper}

Please note that where the full-text provided on Manchester Research Explorer is the Author Accepted Manuscript or Proof version this may differ from the final Published version. If citing, it is advised that you check and use the publisher's definitive version.

\section{General rights}

Copyright and moral rights for the publications made accessible in the Research Explorer are retained by the authors and/or other copyright owners and it is a condition of accessing publications that users recognise and abide by the legal requirements associated with these rights.

\section{Takedown policy}

If you believe that this document breaches copyright please refer to the University of Manchester's Takedown Procedures [http://man.ac.uk/04Y6Bo] or contact uml.scholarlycommunications@manchester.ac.uk providing relevant details, so we can investigate your claim.

\section{OPEN ACCESS}




\section{On Evaluating the Performance of Intentional Controlled Islanding Schemes}

\author{
Jairo Quirós-Tortós, Student Member, IEEE \\ The University of Manchester \\ School of Electrical \& Electronic Engineering \\ jairoquirostortos@ieee.org \\ Mathaios Panteli, Student Member, IEEE \\ The University of Manchester \\ School of Electrical \& Electronic Engineering \\ mathaios.panteli@manchester.ac.uk
}

\author{
Vladimir Terzija, Senior Member, IEEE \\ The University of Manchester \\ School of Electrical \& Electronic Engineering \\ terzija@ieee.org \\ Peter A. Crossley, Member, IEEE \\ The University of Manchester \\ School of Electrical \& Electronic Engineering \\ peter.crossley@manchester.ac.uk
}

\begin{abstract}
Intentional Controlled Islanding (ICI) schemes are used to prevent the total system blackouts that can be caused by large system disturbances, or maloperation of protective relays. Since these schemes are designed to operate when the integrity of the system could not be maintained, their development needs to be accompanied by extensive reliability studies to demonstrate that they fulfill the minimum performance requirements. This would ensure their dependable operation when they have to split the network during the cascading events. The possible causes of an ICI failure to operate as designed have to be determined in order to evaluate their reliability. In this paper, an ICI scheme is firstly presented and illustrated using the IEEE 9-bus test system. Next, a generic procedure for assessing the reliability of ICI schemes is discussed. This procedure and its analysis demonstrate the possible sources of ICI incorrect operation and provide the guidelines for estimating the probability of such undesirable events.
\end{abstract}

Index Terms--Fault tree analysis, controlled islanding, Markov modeling, phasor measurement units, reliability assessment.

\section{INTRODUCTION}

Intentional Controlled Islanding (ICI) schemes are used to prevent the cascading outages and total system blackouts that can be caused by large system disturbances. When the power system integrity could not be maintained, splitting actions are carried out to separate the system into smaller stable subsystems, also known as islands [1-5].

Reference [6] states that ICI methods can be visualized as a contingency dependant/event based schemes. This conclusion is drawn under the consideration that ICI schemes use local information, as well as relevant remote sites, and send the data to a processing location to counteract the propagation of major disturbances in the system. Considering this, ICI schemes can, therefore, be classified as System Integrity Protection Schemes (SIPS) [7]. However, current ICI schemes are more active i.e. their operation cannot be predicted, and their response is based on certain system conditions.

This work was supported in part by the Engineering and Physical Science Research Council (EPSRC) in the UK and The University of Costa Rica.
The main objective during ICI is to determine the set of lines that needs to be disconnected across the network in order to create electrically isolated and stable islands. This must be determined considering minimum possible load-generation mismatches within islands, ensuring generator coherencies and other static and dynamic constraints [1-5].

Several ICI methods have been proposed in the past [1-5]. However, all these methods rely on the functionality of the elements involved in the controlled separation. In other words, all these methods have determined the splitting strategy and the possible sources of ICI incorrect operation have been neglected. Neglecting these inherent sources of errors might eventually create unbalanced islands, and therefore, it might aggravate the problems caused by the cascading events. Consequently, these errors might rapidly bring the power system to the high-speed cascading stage of the blackout.

Since ICI schemes are commonly triggered when the system integrity could not be maintained, their failure to operate would result in very severe consequences. Therefore, it is of great interest to assess the reliability of existing ICI methods in order to evaluate their performance in terms of their dependability and investigate how these schemes can be improved. It is important to note that ICI methods cannot be always armed. These schemes will operate only if particular conditions in the perturbed system are obtained.

In this study, a generic procedure for reliability assessment of ICI schemes is presented. Markov modeling [7], [8] and Fault Tree Analysis (FTA) [7], [8] are suggested for this purpose. Synchronized Measurement Technology (SMT) is considered in this paper. The scenario at which installed Phasor Measurement Units (PMUs) fail to activate the ICI scheme is also evaluated. An illustrative example using the IEEE 9-bus test system is presented in detail. This system will also be used to implement the generic procedure and evaluate the reliability of ICI schemes.

The paper is organized as follows. Section II presents an ICI scheme, the potential use of SMT for ICI and a numerical example of an ICI scheme. Section III introduces the generic 
procedure to evaluate the reliability of ICI schemes, and presents an illustrative example of the reliability assessment of ICI schemes. The conclusions drawn from the study and future work are finally provided in Section IV.

\section{ICI SCHEMES AND SMT}

\section{A. Intentional Controlled Islanding}

ICI methods are used to cope with different extreme system conditions, such as un-damped oscillations, voltage collapse, cascading trips, etc. To date, ICI methods have relied on the operability of the elements involved in the controlled separation. Nevertheless, as the controlled separation is commonly applied as the last resort to avoid complete blackouts, it is of great interest that the splitting actions are effectively implemented when these must split the power system into balanced islands. Thus, it is vital to assess the reliability of existing ICI methods in order to evaluate their performance in terms of their dependability and investigate how these schemes can be improved.

ICI can be included in the wide range of SIPS. SIPS are installed to protect the integrity of power systems or strategic portions thereof, as opposed to conventional protection systems that are dedicated to a specific power system element [9]. ICI methods are commonly applied after observing that in multi-machine power systems groups of generators tend to oscillate against each other after large disturbances.

The existing ICI methods can be grouped according to the objective function they use: a) minimal dynamic coupling [1], b) minimal power imbalance [2-4], or c) minimal power-flow disruption [5], [10]. After a large disturbance, which caused the creation of $r$ coherent groups of generators, one approach is to create auto-sufficient islands with reduced loadgeneration imbalance. This characteristic will also be beneficial during the restoration process [3].

In practical power network, it is commonly assumed that local reactive power compensators can balance the reactive power. The real power balance and real power-flow in each island are more important after system splitting. Assuming a lossless network, the minimum load-generation imbalance within each island can be formulated as follows.

$$
\min _{\forall i \in \mathbf{V}_{\sigma}}\left(\sum_{k} P_{G, k}^{i}-\sum_{l} P_{L, l}^{i}\right)
$$

where $P_{G, k}^{i}$ and $P_{L, l}^{i}$ are the active power of the generator $k$ and load $l$ within island $\sigma=1,2, \ldots, r$, respectively. $\mathbf{V}_{\sigma}$ represents the set of nodes in the island $\sigma$.

Since the aim of this paper is to state the need to evaluate the performance of existing ICI methods, the balanced loadgeneration criterion will be considered. However, any other objective function can also be applied. Therefore, after a large disturbance which causes the creation of coherent groups of generators, the optimal set of branches to be disconnected will be determined with the objective of minimizing the loadgeneration imbalance within the created islands.

\section{B. Syncrhonized Measurement Technology}

Synchronized measurement technology, represented by phasor measurement units, provides access to voltage and current phasors [11]. SMT provides efficient solutions to many problems in modern power systems. In this work, PMUs are expected to be installed across future power systems. Thus, it is assumed that sufficient information will be available to monitor and to support during the controlled separation. The information obtained from SMT can eventually be used in future power systems to determine the coherent groups of generators [12], to monitor the power flow in the transmission lines, to army ICI methods, and to support on determining the optimal splitting strategy.

An Integer Linear Programming (ILP) formulation, which takes advantage of zero injection buses, is used here to allocate PMUs across the power system. Without loss of generality, it is assumed that every PMU has a sufficient number of channels to measure the current phasors through all the branches incident to the corresponding PMU buses (i.e. the busses in which PMUs are installed). This paper does not consider conventional measurements; it is rather a pure PMU based solution, taking the advantage of unique high reporting rates possessed by the modern PMUs.

A binary variable vector $\mathbf{y}$, which represents the presence of PMUs across the power system, is defined. Considering this, the $i$-th entry of $\mathbf{y}$ is defined as:

$$
y_{i}= \begin{cases}1, & \text { if a PMU is placed at boundary bus } i \\ 0, & \text { otherwise }\end{cases}
$$

For an $n$-bus system, the PMU placement problem can be formulated as follows:

$$
\min \sum_{i=1}^{n} c_{i} \cdot y_{i}
$$

subject to,

$$
f(\mathbf{Y}) \geq\left[\begin{array}{llll}
1 & 1 & \cdots & 1
\end{array}\right]_{n \times 1}^{T}
$$

where $c_{i}$ is the cost of the PMU installed at bus $i, f(\mathbf{Y})=\mathbf{B} \times \mathbf{y}$ and $\mathbf{B}=\left[b_{i j}\right]$ is the network connectivity matrix defined as follows:

$$
b_{i j}= \begin{cases}1, & \text { if } i=j \\ 1, & \text { if } i \text { and } j \text { are connected } \\ 0, & \text { otherwise }\end{cases}
$$

Even though the presented formulation determines the optimal PMU placement for observability analysis, it is assumed in this paper that real-time monitors are also installed at all the terminal buses of synchronous generators. This information will be used to determine the coherent groups of generators after large disturbances [12].

\section{Numerical Illustration of the ICI Scheme}

The IEEE 9-bus test system, shown in Fig. 1, is used in this paper as illustrative example of the mentioned ICI scheme. It will also be used to point out the need to assess the reliability of ICI schemes in the next section. The IEEE 9-bus test system has 3 synchronous generators, 9 branches (6 transmission lines and 3 transformers) and 3 constant power loads [13]. It is assumed that the rated power flow in the transmission lines is $100 \mathrm{MVA}$. This information is vital since 
it is well known that overloads in transmission lines can cause cascading events due to the tripping of lines by local protection devices. This information will be used in this paper to activate the scheme when transmission lines exceed their rated capacity by $50 \%$.

By implementing the presented PMU placement method described in Section II-B, it is concluded that two devices are needed in order to have complete observability and to monitor the power system. As shown in Fig. 1, these PMUs are located at buses 4 and 8 . It is also considered that each generator is equipped with real-time monitors. As previously mentioned, these devices are used to determine the coherent groups of generators, to monitor the power flow in the transmission lines, to army the ICI method, and to support on determining the optimal splitting strategy.

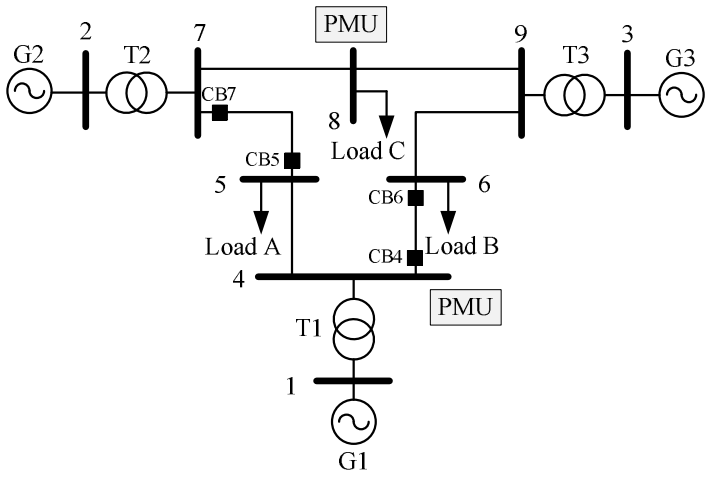

Figure 1. Single line diagram of the IEEE 9-bus test system

Suppose that at $t=0.20 \mathrm{~s}$, a three-phase fault occurs on line 5-7 near to bus 5 of the IEEE 9-bus system. This disturbance is cleared after local relays open the line at $t=0.45 \mathrm{~s}$. This disconnection is actually done by opening the Circuit Breakers (CBs) at bus 5 (CB5) and 7 (CB7). In Fig. 2, it can be observed that after the fault is cleared, two groups of coherent generators are created. Group 1 consists of G2 and G3, and Group 2 consists of only G1.

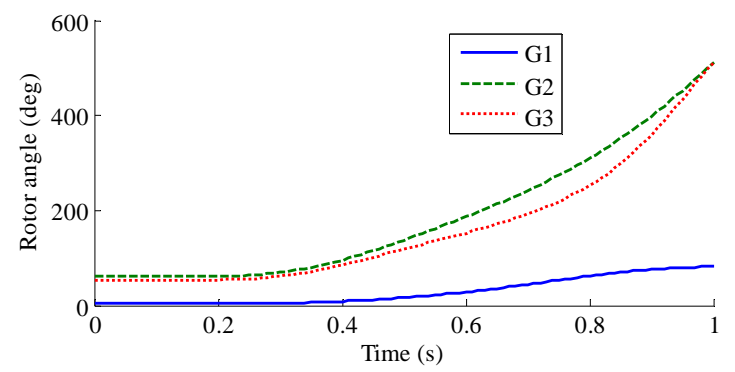

Figure 2. Rotor angles of the three generator in the IEEE 9-bus test system

For this particular example, it can be seen in Fig. 1 that two possible splitting strategies can be implemented. Since transmission line 5-7 is disconnected, the islands can be created by disconnecting lines 4-6 or 6-9. Considering these two possible splitting strategies, Table I presents the active power imbalance within each island.

As noticed in Table I, when splitting the system across the transmission line 4-6, the load-generation imbalance within each island is smaller than the second case i.e. when splitting the system across line 6-9. Since the load-generation imbalance is smaller in the first case, it is imperative to split the system by intentionally disconnecting line 4-6 when all the conditions are satisfied i.e. when two coherent groups of generators are obtained and when the power flow in line 6-9 overreaches by $50 \%$ its rated capacity.

TABLE I. Results For The Two Possible SPlitTing STRATEGIES

\begin{tabular}{cccc}
\hline \hline $\begin{array}{c}\text { Line to be } \\
\text { disconnected }\end{array}$ & $\begin{array}{c}\text { Island } \\
\text { No. }\end{array}$ & $\begin{array}{c}\text { Buses within } \\
\text { islands }\end{array}$ & $\sum_{k} P_{G, k}^{i}-\sum_{l} P_{L, l}^{i}$ \\
\hline \hline \multirow{2}{*}{$4-6$} & 1 & $1,4,5$ & -53.36 \\
& 2 & $2,3,6,7,8,9$ & 58.00 \\
\hline \multirow{2}{*}{$6-9$} & 1 & $1,4,5,6$ & -143.36 \\
& 2 & $2,3,7,8,9$ & 148.00 \\
\hline \hline
\end{tabular}

Applying the first splitting strategy, it can be noticed in Fig. 3 that the frequency in the Island 2 increases and the same value decreases in the Island 1. Fig. 4 also shows these features, but when the second splitting strategy is applied. Comparing Figs. 3 and 4, it can be noted that frequency in the island 1 decreases smoother when applying the first splitting strategy. Similarly, the frequency in the island 2 increases less rapidly when splitting the system across line 4-6.

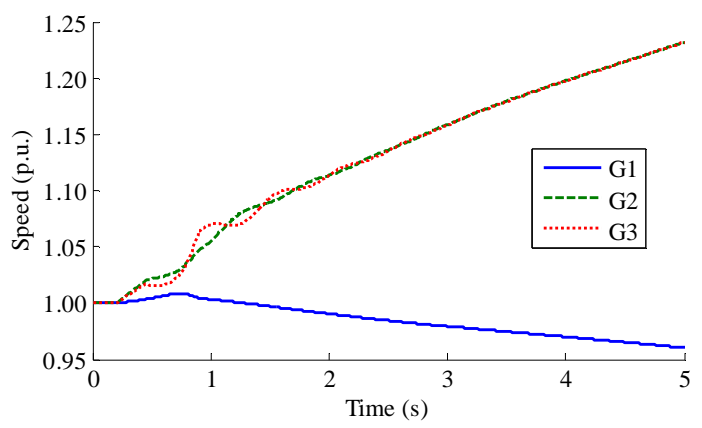

Figure 3. Frequency in the islands when splitting the system across line 4-6

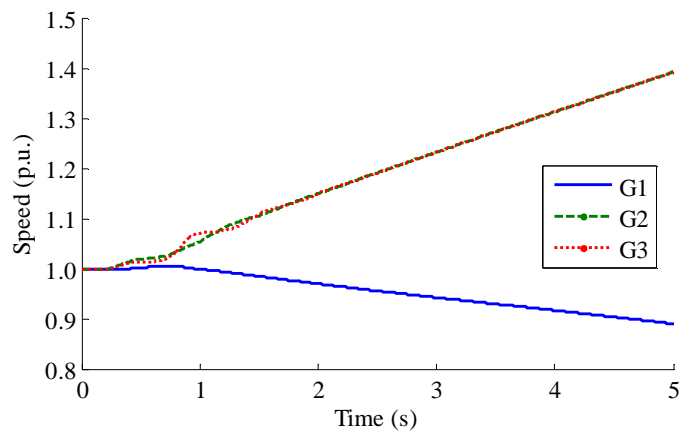

Figure 4. Frequency in the islands when splitting the system across line 6-9

Implementing the first splitting strategy produces smaller load-generation imbalance within islands. Therefore, reduced load shedding and generation shedding are required after the controlled separation. Considering the slow motion of secondary controls, it is possible to return both frequencies to the nominal value.

\section{GENERIC PROCEDURE FOR RELIABILITY ASSESSMENT OF INTENTIONAL CONTROLLED ISLANDING SCHEMES}

In general, an ICI operation can be classified into the following three categories:

1) desirable operation;

2) undesirable operation; 
3) failure to operate.

An ICI operation can be desirable or undesirable, depending on whether or not the consequence of the ICI operation is preferred over the consequence of not ICI operation. On the other hand, an ICI scheme failure to operate occurs when the ICI scheme fails to split the power system into islands. This failure to operate can be caused by several reasons, such as hardware and software failures, faulty design logic, and human error. Therefore, a quantitative analysis to ensure that the system could meet the reliability requirements is important and necessary.

\section{A. Description of Procedure}

There are several methods to assess the reliability of ICI schemes. Aforementioned, the operation of ICI schemes is similar to the operation of SIPS. Reference [7] describes different methods for assessing SIPS reliability. In this paper, Markov modelling and Fault Tree Analysis are used, as illustrated in Fig. 5. A detailed description of these methods can be found in [7], [8] and [14].

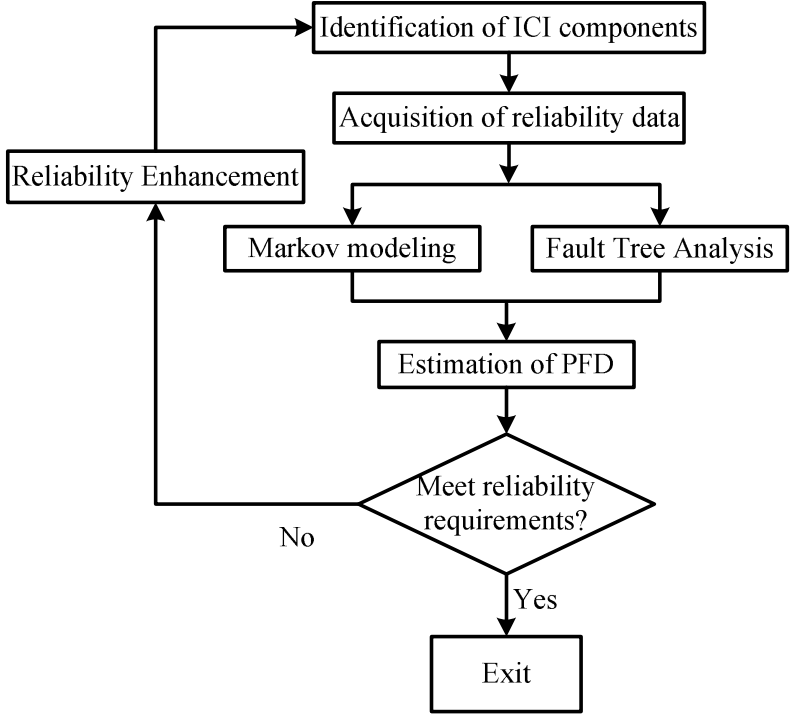

Figure 5. Generic procedure to assess the reliability of ICI schemes

The first step is identifying the components involved in the ICI operation. Given the previous analysis, the scheme logic can be seen in Fig. 6, where the ICI components can be determined. FTA is a simple way of representing the operation of ICI schemes, and in general the operation of event-based schemes. As noticed, the "Arming Signal" is received from CB7 or CB5. These signals, in addition with the "Activation Signal" will produce the CBs at bus 4 (CB4) and 6 (CB6) to trip. The scheme logic operations are performed by logic processors or Programmable Logic Controllers (PLC). As shown in Fig. 6, the "Activation Signal" is obtained when the line 6-9 overreach by $50 \%$ its rated capacity. In real power systems, the "Arming Signal" is expected to be obtained by analysing the status of CBs. On the other hand, the "Activation Signal" will be received from installed PMUs. These devices will monitor the power flow in the lines and when the power flow through the line reaches $150 \mathrm{MW}$, the control actions will be carried out.

The next step is gathering the reliability data of the ICI components. The necessary data for carrying out the reliability studies are the failure and repair rates of the ICI components. Then, Markov modelling is used to estimate the state probabilities of the components at any given time. Numerous states can be considered in the Markov models, such as failure to operate or operation when not required. These state probabilities are then inserted in the fault trees for estimating the probability of the scheme failing to operate when required. The fault trees provide the events or combination of events that can result in an ICI misoperation.

After estimating the overall probability of failure on demand (PFD), it must be determined if the scheme meets the minimum reliability requirements. If not, reliability enhancement must be applied, such as redundancy, reduce the time interval of the scheme testing and maintenance, or logic modification.

Since the objective of this paper is to state the need to assess the reliability of ICI schemes, the complete analysis of this method is not given in this paper. Instead, a simple illustrative example is presented in Section III-B to demonstrate the application of this method on the ICI scheme described and illustrated in Section II. For future work, more reliability studies for identifying the most critical components in ICI operation will be performed. A more detailed and accurate reliability assessment, taking into account the communications reliability, should be carried out for real case applications.

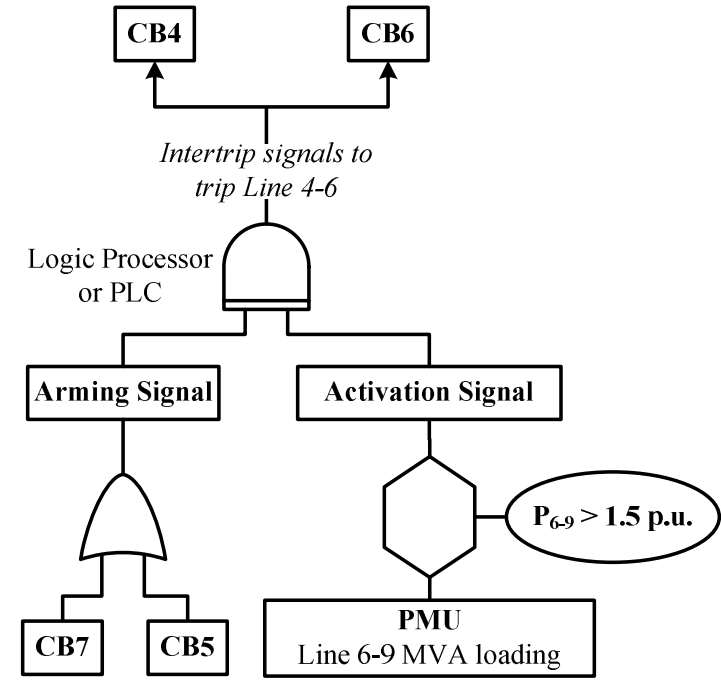

Figure 6. Scheme operating logic of the ICI scheme

\section{B. Illustrative Example}

The fault tree providing the events that can lead to the PFD of the ICI method described in this paper is presented in Fig. 7. It can be seen that there are four possible sources of errors. If the arming signal (trip of CB5 or CB7) is not received, the scheme will fail to operate when needed. PMUs are also possible source of errors. Here, the communications channels play an important role. These are critically important to assess the reliability of ICI schemes. As shown, the loss of these channels will not activate the ICI scheme. The lack of the scheme activation will eventually produce cascading events and might lead to the creation of unbalanced islands. Even though it has not been discussed in this paper, logic processors and PLCs can contribute to the scheme failure to 
operate. These failures are considered as software or hardware failures and must be evaluated when assessing the reliability of ICI schemes. Provided that the arming and activation signals are transmitted and received correctly and timely, the implementation of the actions can also introduce problems to ICI schemes. As noticed in Fig. 7, the failure to split the system can also produce the scheme failure to operate.

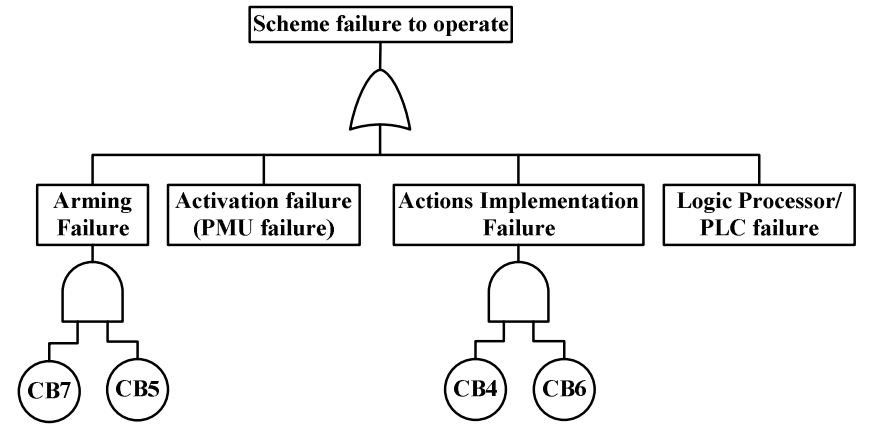

Figure 7. Fault tree of the ICI scheme

TABLE II. COMPONENTS RELIABILITY DATA

\begin{tabular}{cc}
\hline Component & PFD (per year) \\
\hline \hline CBs & 0.01 \\
PMU & 0.0026 \\
PLC & 0.0033 \\
\hline \hline
\end{tabular}

Assuming that the application of the Markov modelling gives the components PFD shown in Table II after one year, the overall PFD of the ICI scheme can be evaluated using the following equations:

$$
\begin{gathered}
P F D_{\text {total }}=P F D_{\text {Arming }}+P F D_{\text {Activation }} \\
+P F D_{\text {Implementation }}+P F D_{P L C} \\
P F D_{\text {Arming }}=P F D_{C B 7} \times P F D_{C B 5} \\
P F D_{\text {Implementation }}=P F D_{C B 6} \times P F D_{C B 4}
\end{gathered}
$$

Applying the components PFD of Table II in (6)-(8), the overall PFD of the scheme is equal to $6.1 \times 10^{-3}$. Then, it must be investigated if the estimated PFD meets the minimum reliability requirements. If not, reliability improvement has to be applied, as described in the previous section.

\section{CONCLUSIONS AND FUTURE WORK}

Intentional Controlled Islanding methods can effectively support system operators to reduce the impact of large scale power system blackouts. ICI schemes have been considered as dependant/event-based schemes. However, since the actual splitting strategy depends on the current network topology during the cascading events, it is difficult in real systems to determine in advance where the network will be actually split. Thus, these schemes should be more active i.e. these should be considered response-based schemes. As demonstrated in this paper, incorrect system separation might create unbalanced islands, increasing the amount of load to be shed and the generation to be rescheduled. On the other hand, correct splitting actions can maintain the frequency in the islands within acceptable limits and help during the restoration process.
Existing ICI schemes have relied on the functionality of the elements involved in the controlled separation. Nevertheless, the elements involved in the ICI scheme can fail, causing the probability of failure on demand to increase. Therefore, it is of great interest for the reliable operation of the whole network to assess the reliability of existing ICI methods. The aim of these reliability studies should be the evaluation of their performance in terms of their dependability and the investigation of how these schemes can be improved. By evaluating the performance of ICI schemes, it is possible to determine the possible sources of errors, and therefore, different refinements/improvements can be implemented. Redundancy, voting schemes, decrease of the schemes' testing time interval and refinement of the logic of ICI schemes are possible ways to enhance the reliability of these schemes.

This paper evaluated the performance of an ICI scheme. Minimum load-generation imbalance was considered during the controlled separation. However, it is vitally important to evaluate the impact of each ICI method on the overall PFD. Also, it is vital to run more reliability studies for identifying the most critical components in ICI operation. A more detailed and accurate reliability assessment, taking into account the communications reliability (such as communication processors and links), should be carried out for real case applications. This is of great interest as these schemes are heavily dependent on fast and reliable communications.

\section{REFERENCES}

[1] S.B. Yusof, G.J. Rogers, and R.T.H. Alden, "Slow coherency based network partitioning including load buses," IEEE Trans. Power Syst., vol. 8, no. 3, pp. 1375-1382, Aug. 1993.

[2] K. Sun, D. Zheng, and Q. Lu, "Splitting strategies for islanding operation of large-scale power systems using OBDD-based methods," IEEE Trans. Power Syst., vol. 18, no. 2, pp. 912-922, May 2003.

[3] H. You, V. Vittal, and X. Wang, "Slow coherency - Based islanding," IEEE Trans. Power Syst., vol. 19, no. 1, pp. 483-491, Feb. 2004.

[4] G. Xu and V. Vittal, "Slow coherency based cutset determination algorithm for large power systems," IEEE Trans. Power Syst., vol. 25, no. 2, pp. 877-884, May. 2010.

[5] L. Ding, F. Gonzalez-Longatt, P. Wall, and V. Terzija, "Two-step spectral clustering controlled islanding algorithm," IEEE Trans. Power Syst., vol. 28, no. 1, pp. 75-84, 2013.

[6] PSERC, "System Protection Schemes: Limitations, Risks, and Management," Dec. 2010

[7] J.D. McCalley and W. Fu, "Reliability of Special Protection Schemes," IEEE Trans. Power Syst., vol. 14, no. 4, pp. 1400-1406, Nov. 1999.

[8] T.Y. Hsiao, C.A. Shieh, and C.N. Lu, "Reliability evaluation of a Taipower system protection scheme," in IEEE PES Power Systems Conference and Exposition, 2004, pp. 157-162.

[9] V. Madani, et al., "IEEE PSRC Report on Global Industry Experiences With System Integrity Protection Schemes (SIPS)," IEEE Trans. Power Del., vol. 25, no. 4, pp. 2143-2155, Oct. 2010.

[10] J. Quiros Tortos and V. Terzija, "Controlled Islanding Strategy Considering Power System Restoration Constraints," in IEEE PES General Meeting, San Diego, 2012, pp. 1-8.

[11] V. Terzija, et al., "Wide-area monitoring, protection, and control of future electric power networks," Proceedings of the IEEE, vol. 99, pp. 80-93, Jan. 2011.

[12] M. Jonsson, M. Begovic, and J. Daalder, "A new method suitable for real-time generator coherency determination," IEEE Trans. Power Syst., vol. 19, no. 3, pp. 1473-1483, Aug. 2004.

[13] P.M. Anderson and A.A. Fouad, Power System Control and Stability, $2^{\text {nd }}$ ed. New York: IEEE Press, 2003.

[14] U.S. Nuclear Regularoty Commission, "Fault Tree Handbook," Jan. 1981. 